\title{
3D Reconstruction of Dislocation Structures in $\mathrm{Fe}-\mathrm{Al}_{28,40}$
}

\author{
J. VESELÝ ${ }^{a, *}$ AND J. KOPEČEK ${ }^{b}$ \\ ${ }^{a}$ Charles University in Prague, Department of Physics of Materials, Ke Karlovu 5, 12116 Prague 2, Czech Republic \\ ${ }^{b}$ Academy of Sciences of the Czech Republic, Institute of Physics, Prague, Czech Republic

\begin{abstract}
Some of the experimental problems faced when attempting dislocation tomography are discussed. A method of $3 \mathrm{D}$ reconstruction of straight line segments with much lower experimental requirements than tomography is proposed. This method is applied to dislocation structures found in two iron aluminium alloys (28 and 40 at.\% Al). Resulting information on statistical distribution of dislocation segment lengths and orientations is presented.
\end{abstract}

DOI: $10.12693 /$ APhysPolA.128.787

PACS: 68.37.Lp, 61.72.Ff

\section{Introduction}

Oftentimes it is of interest to describe the dislocation structures in three dimensions. Historically stereo pairs and anaglyphs have been used to convey some sense of 3D. However, these provide a view from only one direction with a limited impression of depth. More recently tomography methods were successfully applied to dislocations $[1,2]$. This approach is experimentally challenging even with state of the art equipment.

The theory of tomographic reconstruction relies on the fulfillment of projection requirement [3]. It requires that the image intensity is a monotonous function of sample thickness (and at most one other material characteristic). This criterion is fulfilled for mass-thickness contrast, indeed tomographic reconstruction is routinely used on biological specimens. Material science is more interested in composition dependent signals such as STEM-HAADF "Z-contrast", energy filtered images or EDX maps that also fall in this category [3]. On the other hand, in order to image dislocations, diffraction contrast is needed. Diffraction contrast depends strongly on the orientation and thus it does not fulfill the projection requirement. Nevertheless the tomographic reconstruction of weak beam dark field (WBDF) images was successful [1]. The process, though, was very difficult, since the diffraction condition had to be aligned exactly during the tilt series acquisition. Later STEM-ADF signal was used with convergent beam mitigating the need for exact alignment $[2,4]$.

In theory, the tomographic reconstruction requires infinite number of projections over full $180^{\circ}$. In practice one image every $2^{\circ}$ over $120-140^{\circ}$ is often considered adequate. This is usually impossible to achieve with standard holders and therefore specialized high tilt tomography holders are available. However these often lack the flexibility needed to align the diffraction conditions. Therefore high angle triple axis holder had been designed

*corresponding author; e-mail: vesely@gjh.sk specifically for the dislocation tomography [5]. The need for constant diffraction conditions also makes it difficult to apply dislocation tomography to phenomena occurring across grain boundaries unless the grains are in special orientation relationship [6].

The result of the tomographic reconstruction is the $3 \mathrm{D}$ map of intensity, where the dislocations are often blurred due to the wedge missing from the tilt series, alignment problems and diffraction effects. Therefore these clouds of intensity have to be interpreted as 1D dislocation lines for example by tracing the ridge lines [6].

In this work, a less demanding method is developed. Only a handful of projections (at least two) are needed to reconstruct dislocation geometry if its one dimensional nature is considered. The method of stereographic reconstruction of straight dislocation segments is briefly presented and then applied to two dislocation structures observed in iron aluminum intermetalic alloys.

\section{Method}

Dislocations are modeled as straight lines between points recognizable on multiple projections such as junctions and intersections with foil surfaces. Coordinates of these points when projected from 3D space are given by

$$
\left(\begin{array}{lll}
R_{X x}^{j} & R_{X y}^{j} & R_{X z}^{j} \\
R_{Y x}^{j} & R_{Y y}^{j} & R_{Y z}^{j}
\end{array}\right)\left(\begin{array}{c}
x_{i} \\
y_{i} \\
z_{i}
\end{array}\right)+\left(\begin{array}{c}
o_{x}^{j} \\
o_{y}^{j}
\end{array}\right)=\left(\begin{array}{c}
X_{i}^{j} \\
Y_{i}^{j}
\end{array}\right),
$$

where $R_{a b}^{j}$ are the elements of $j$-th projection rotation matrix, $\left(x_{i}, y_{i}, z_{i}\right)$ are $3 \mathrm{D}$ coordinates of $i$-th point, $\left(X_{i}^{j}, Y_{i}^{j}\right)$ are 2D coordinates of $i$-th point in $j$-th projection and $\left(o_{x}^{i}, o_{y}^{i}\right)$ are the offsets of the origin of image of $j$-th projection. When assembled for each point observed in every projection $\left(X_{i}^{j}, Y_{i}^{j}\right)$ this results in a system of linear equations for unknowns $\left(x_{i}, y_{i}, z_{i}\right)$ and $\left(o_{x}^{i}, o_{y}^{i}\right)$. To fix the origin of a coordinate system three more equations are needed:

$$
\sum_{i} x_{i}=\sum_{i} y_{i}=\sum_{i} z_{i}=0 .
$$

With the increasing number of projections this system of equations quickly becomes overdetermined and can be 
solved in a least squares sense. The left hand side matrix of the system of linear equations (Eqs. (1) and (2)) is very sparse and the problem can therefore be solved efficiently even for a large number of points and projections.

In principle, the rotation matrix for a given projection can be determined from the goniometer angles. However, in our case the goniometer was not precise enough and the orientation was determined from the Kikuchi line patterns with the accuracy of about $0.3^{\circ}$. Another advantage of using the Kikuchi patterns is that the final coordinate system is directly referenced to the crystal lattice.

This approach is similar to Karcher and Robertson [7], who placed markers at dislocation intersections with surfaces. However, they used tomography instead of algebraic equations to reconstruct the marked positions and therefore still required whole tilt series.

\section{Results}

JEOL JEM-2000FX with analytical double tilt holder was used to acquire projection images. First example is iron aluminium intermetallic with 28 at.\% aluminium. Single crystal was deformed in compression to about $3 \%$ strain at $900 \mathrm{~K}$. The network of interacting dislocations was observed. Collages of bright field images were taken in five orientations close to the limits of goniometer range (Fig. 1a) and the method described in previous section was used to create a $3 \mathrm{D}$ model (note that $\mathrm{Fe}-\mathrm{Al}_{28}$ is magnetic and tilt series acquisition would require constant realignment of a microscope). Figure 2a shows the projection of the 3D model back on one of the observed collages. Dislocation segments are colored according to their directions (Fig. 3a). The thickness of the specimen and large number of dislocation segments precluded the Burgers vector analysis for the whole network. However, some of the longer blue segments were identified by invisibility criteria to be the pairs of screw dislocations. In thinner parts of the specimen the interconnectedness of the dislocation network is not evident.

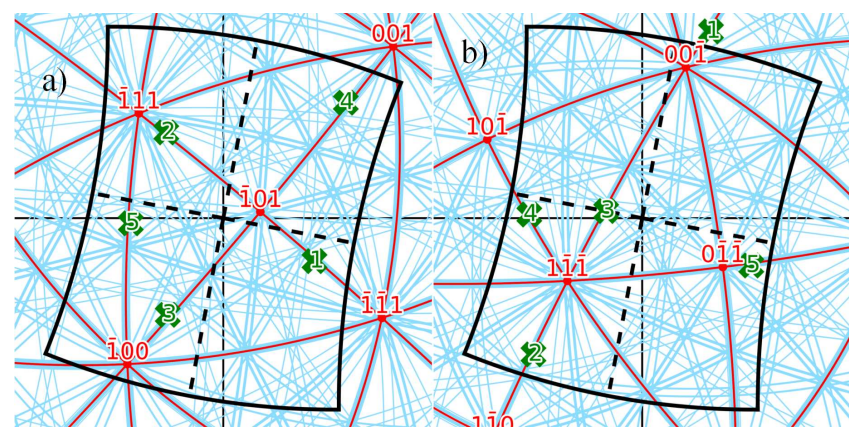

Fig. 1. Kikuchi maps of observed crystals: a) $\mathrm{Fe}-$ $\mathrm{Al}_{28}$, b) $\mathrm{Fe}-\mathrm{Al}_{40}$. Numbered marks show 5 orientations used for reconstruction, approximate range of double tilt holder is shown as distorted rectangle.

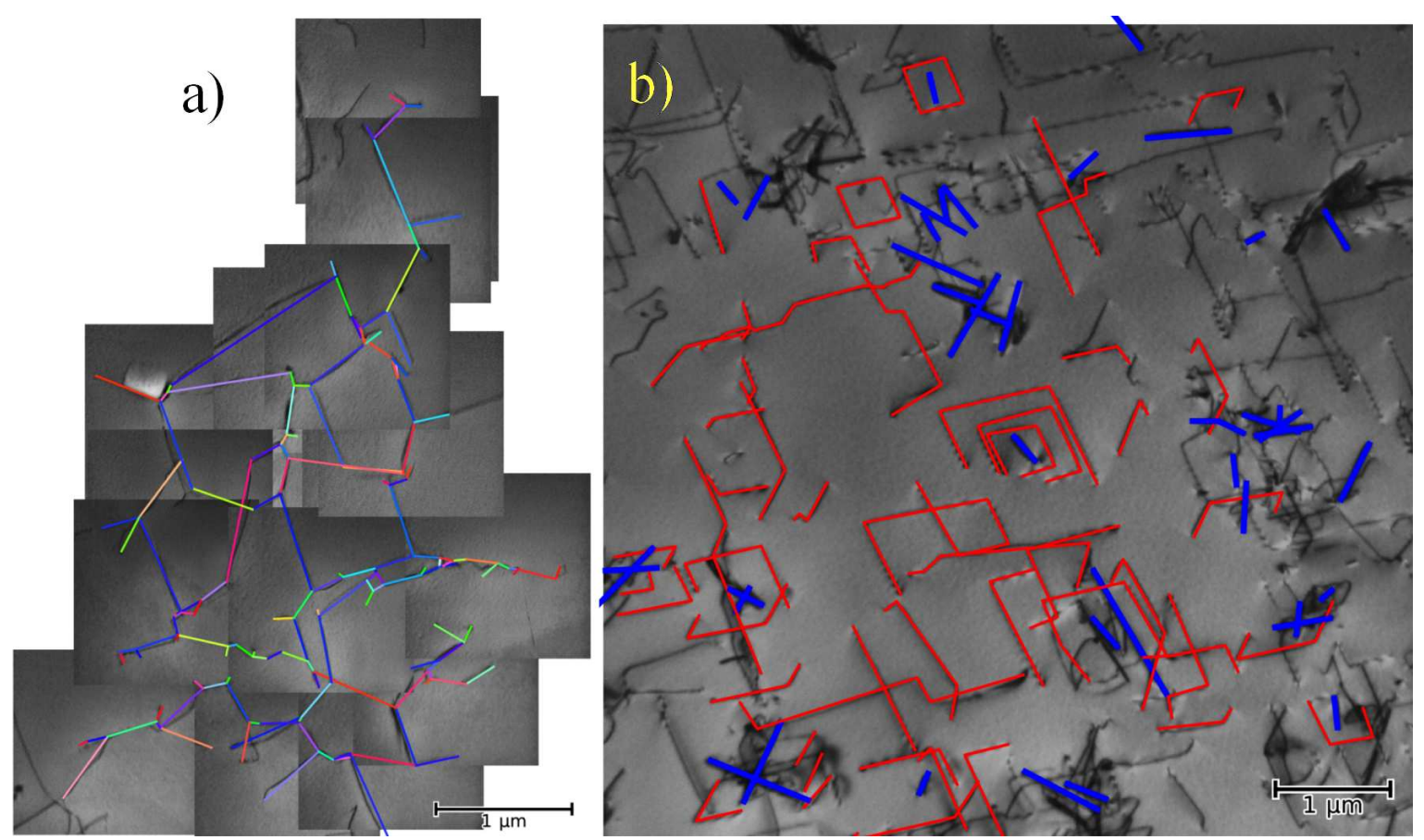

Fig. 2. Images used to reconstruct the 3D models taken in both cases in orientation \#3 in Fig. 1. Images are overlaid with a projections of $3 \mathrm{D}$ models: a) $\mathrm{Fe}-\mathrm{Al}_{28}$ line colors are based on segment direction (Fig. $3 \mathrm{a}$ ), b) $\mathrm{Fe}-\mathrm{Al}_{40}$ dislocations are shown in red, particles are blue. 


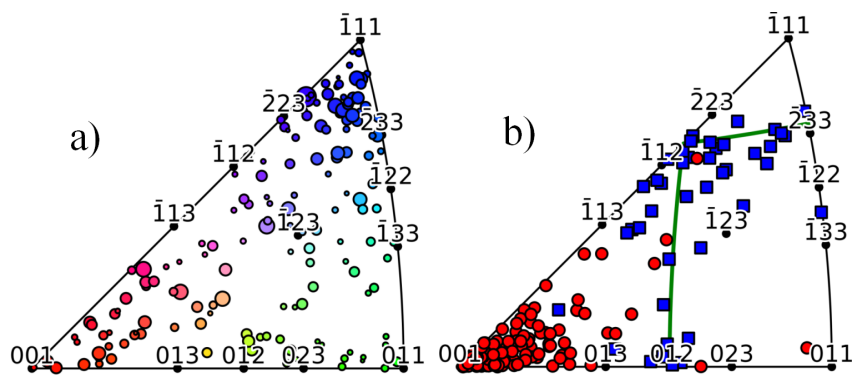

Fig. 3. Symmetry reduced pole figures showing directions extracted from 3D models. a) segments of dislocation network in $\mathrm{Fe}-\mathrm{Al}_{28}$. Marker size is proportional to segment length. b) dislocation segments and particles in $\mathrm{Fe}-\mathrm{Al}_{40}$. Dislocation segments (red) cluster around [001]. Long axes of particles (blue) cluster around line (green) going approximately from [233] through [112] to [012] as predicted from geometrical lattice match considerations [10].

Second example is iron with 40 at.\% aluminium quenched from $1000^{\circ} \mathrm{C}$ and then annealed for $140 \mathrm{~h}$ at $450^{\circ} \mathrm{C}$. This material is known to retain high concentration of vacancies after quenching [8]. During annealing non equilibrium vacancies condensate on impurity particles $\left(\kappa-\mathrm{Fe}_{3} \mathrm{AlC}\right.$ carbide) and form square loops of edge dislocations around them [9]. Again, bright field images were taken in five orientations shown in Fig. 1b. A model including both dislocation segments and carbide particles was constructed. Its reprojection is shown in Fig. $2 \mathrm{~b}$. Figure $3 \mathrm{~b}$ shows that directions of dislocation segments cluster around $\langle 100\rangle$. Statistics on carbide particles is worse, however non-isotropic distribution of particle axes is evident.

In both examples the mean distance between reprojected and measured points is about $20 \mathrm{~nm}$. As can be seen in Fig. 2 the reconstructed models represent the geometry of observed dislocation structures quite well, despite the rather crude assumption of straight dislocation segments.

\section{Summary}

$3 \mathrm{D}$ reconstruction of straight line segments with much lower experimental requirements than tomography was implemented. Resulting 3D models can be visualized and interactively examined on computer. Additionally interesting statistical information on distributions of orientations (Fig. 3) and segment lengths can be obtained. Presented approach can be extended to curved dislocations by including the control points along the dislocation line. In that case measurements of curvature would provide direct information on acting local stresses. Slip planes of curved dislocation could be determined as well. Dislocation structure models in the presented form correspond directly to the dislocation dynamics models and could be used as realistic initial conditions.

\section{Acknowledgments}

The present work is a part of the Czech Grant Agency project $14-36566 \mathrm{G}$.

\section{References}

[1] J.S. Barnard, J. Sharp, J.R. Tong, P.A. Midgley, Science 313, 319 (2006).

[2] M. Tanaka, M. Honda, M. Mitsuhara, S. Hata, K. Kaneko, K. Higashida, Mater. Trans. 49, 1953 (2008).

[3] P.A. Midgley, M. Weyland, Ultramicroscopy 96, 413 (2003).

[4] J.H. Sharp, J.S. Barnard, K. Kaneko, K. Higashida, P.A. Midgley, J. Phys. Conf. Ser. 126, 012013 (2008).

[5] S. Hata, H. Miyazaki, S. Miyazaki, M. Mitsuhara, M. Tanaka, K. Kaneko, K. Higashida, K. Ikeda, H. Nakashima, S. Matsumura, J.S. Barnard, J.H Sharp, P.A. Midgley, Ultramicroscopy 111, 1168 (2011).

[6] J.P. Kacher, G.S. Liu, I.M. Robertson, Scr. Mater. 64, 677 (2011).

[7] J. Kacher, I.M. Robertson, Acta Mater. 60, 6657 (2012).

[8] L.M. Pike, I.M. Anderson, C.T. Liu, Y.A. Chang, Acta Mater. 50, 3859 (2002).

[9] M.A. Crimp, B.A. Simkin, B.C. Ng, Philos. Mag. Lett. 81, 833 (2001).

[10] J. Veselý, Ph.D. Thesis, Charles University in Prague, 2015 . 